\title{
Mapping White Matter Integrity and Neurobehavioral Correlates in Children with Fetal Alcohol Spectrum Disorders
}

\author{
Elizabeth R. Sowell, ${ }^{1}$ Arianne Johnson, ${ }^{1}$ Eric Kan, ${ }^{1}$ Lisa H. Lu, ${ }^{1}$ John Darrell Van Horn, ${ }^{1}$ Arthur W. Toga, ${ }^{1}$ \\ Mary J. 0'Connor, ${ }^{2}$ and Susan Y. Bookheimer ${ }^{2}$ \\ ${ }^{1}$ Laboratory of Neuro Imaging, Department of Neurology, and ${ }^{2}$ Department of Psychiatry and Biobehavioral Sciences, University of California, Los Angeles, \\ Los Angeles, California 90095-7334
}

\begin{abstract}
Brain structural abnormalities and neurocognitive dysfunction have been observed in individuals with fetal alcohol spectrum disorders (FASDs). Little is known about how white matter integrity is related to these functional and morphological deficits. We used a combination of diffusion tensor and T1-weighted magnetic resonance imaging to evaluate white matter integrity in individuals with FASDs and related these findings to neurocognitive deficits. Seventeen children and adolescents with FASDs were compared with 19 typically developing age-and gender-matched controls. Lower fractional anisotropy (FA) was observed in individuals with FASDs relative to controls in the right lateral temporal lobe and bilaterally in the lateral aspects of the splenium of the corpus callosum. White matter density was also lower in some, but not all regions in which FA was lower. FA abnormalities were confirmed to be in areas of white matter in post hoc region of interest analyses, further supporting that less myelin or disorganized fiber tracts are associated with heavy prenatal alcohol exposure. Significant correlations between performance on a test of visuomotor integration and FA in bilateral splenium, but not temporal regions were observed within the FASD group. Correlations between the visuomotor task and FA within the splenium were not significant within the control group, and were not significant for measures of reading ability. This suggests that this region of white matter is particularly susceptible to damage from prenatal alcohol exposure and that disruption of splenial fibers in this group is associated with poorer visuomotor integration.
\end{abstract}

Key words: FAS; FASDs; DTI; VBM; corpus callosum; visuomotor integration

\section{Introduction}

Fetal alcohol syndrome (FAS) results from maternal consumption of alcohol during pregnancy, although it is not clear how much alcohol is required to result in the syndrome. Facial dysmorphology (i.e., smooth philtrum, thin upper lip, small palpebral fissures), growth deficits, and CNS abnormalities are required for the diagnosis of FAS (Jones and Smith, 1975). Children with FAS exhibit a wide range of symptoms including severe growth restriction and intellectual disability. FAS affects $0.5-2$ individuals per 1000 live births across various populations in the United States, but these rates vary by region, race, and culture (National Center on Birth Defects and Developmental Disabilities Centers for Disease Control and Prevention Department of Health and Human Services, 2004). The relatively high incidence

\footnotetext{
Received Sept. 18, 2007; revised Dec. 3, 2007; accepted Dec. 4, 2007.

This work was supported by National Institutes of Health-National Institute on Drug Abuse Grants R21 DA15878 and R01 DA017831, National Institute on Alcohol Abuse and Alcoholism Grants U24AA014808 and U01AA017122, National Center for Research Resources Grants U54 RR021813 and P41 RR013642, and March of Dimes Grant 5FY03-12.

Correspondence should be addressed to Dr. Elizabeth R. Sowell, Laboratory of Neuro Imaging, University of California, Los Angeles, Neuroscience Research Building 1, Room 225, 635 Charles Young Drive South, Los Angeles, CA 90095-7334. E-mail: esowell@loni.ucla.edu.

DOI:10.1523/JNEUROSCI.5067-07.2008

Copyright $\odot 2008$ Society for Neuroscience $\quad$ 0270-6474/08/281313-07\$15.00/0
}

of FAS in the United States and other countries is of considerable social concern given that it is a leading cause of preventable developmental disabilities. Brain abnormalities, most commonly microcephaly and neuronal migration anomalies, have been documented in postmortem studies of FAS (Jones and Smith, 1973; Roebuck et al., 1998). Mental retardation is common among individuals with FAS and subtler neuropsychological abnormalities have also been observed (Mattson and Riley, 1998). Additionally, behavioral and psychosocial problems are frequently observed in these subjects (Roebuck et al., 1999). Children with heavy prenatal exposure to alcohol but without facial dysmorphology required for the FAS diagnosis have also been observed to have neurocognitive (Mattson et al., 1998) and neuroanatomical (Sowell et al., 2001a,b; O'Hare et al., 2005) abnormalities and have been described as falling within a continuum of effects termed fetal alcohol spectrum disorders (FASDs).

Previous brain-imaging studies have shown brain structural abnormalities in children with FASDs (Mattson et al., 1996; Swayze et al., 1997; Riikonen et al., 1999; Sowell et al., 2001b, 2002a,b, 2008). Greater cortical thickness, smaller brain size and less white matter density (WMD) have been observed in parietal and posterior temporal regions (Sowell et al., 2002a, 2008). Less brain activation has been observed in the posterior temporal language regions during a verbal learning task (Sowell et al., 2007) in 
Table 1. Demographics and neurocognitive performance for FASD and control subjects

\begin{tabular}{|c|c|c|c|c|c|c|}
\hline & \multicolumn{2}{|l|}{$\operatorname{FASD}(n=17)$} & \multicolumn{2}{|c|}{ Controls $(n=19)$} & \multirow[b]{2}{*}{$t$} & \multirow[b]{2}{*}{$p$} \\
\hline & Mean & SD & Mean & SD & & \\
\hline Age & 10.525 & 2.714 & 11.238 & 2.805 & 0.775 & 0.444 \\
\hline Handedness & 56.358 & 57.588 & 64.579 & 30.246 & 0.527 & 0.603 \\
\hline Gender & 9 male & & 8 male & & $0.423 \chi^{2}$ & 0.516 \\
\hline Maternal education (years) & 16.706 & 1.687 & 15.474 & 3.893 & -1.206 & 0.236 \\
\hline Ethnicity (\% Caucasian) & $82.35 \%$ & & $36.84 \%$ & & & \\
\hline FSIQ & $88.750(n=16)$ & 14.585 & 109.526 & 17.351 & 3.849 & 0.001 \\
\hline WRAT-RE & 33.765 & 8.899 & 39.947 & 4.983 & 2.531 & 0.018 \\
\hline VMI & 19.824 & 3.729 & 24.000 & 3.873 & 3.294 & 0.002 \\
\hline
\end{tabular}

Mean, $S D, t$, and $p$ values for group differences are given for demographics and neurocognitive tests. We used the $\chi^{2}$ rather than the $t$ test for the group effects on gender.

the FASD relative to the control group, in the same region where greater cortical thickness was seen, suggesting that an abundance of cortical tissue does not provide functional advantage in those with severe prenatal alcohol exposure. Abnormalities of white matter volumes (Archibald et al., 2001) have also been observed in individuals with FASDs. Shape abnormalities have been noted in white matter structures such as the corpus callosum (Bookstein et al., 2001, 2002; Sowell et al., 2001a). Notably, the posterior extent of the corpus callosum, which connects posterior temporal and inferior parietal regions across the left and right hemispheres, appears to be more dysmorphic than anterior regions (Sowell et al., 2001a). Given volume, shape and density abnormalities observed in white matter in individuals with FASDs, further investigation of white matter integrity is warranted.

While it is becoming clear that white matter is particularly vulnerable to prenatal alcohol exposure, white matter microstructure studied with diffusion tensor imaging (DTI) in FASD has only been evaluated in 2 previous reports (Ma et al., 2005; Wozniak et al., 2006), and both reports focused only on the midline corpus callosum. DTI allows measurement of diffusion characteristics of water molecules within brain tissue (for review, see Mori and Zhang, 2006). Anisotropy measures derived from DTI data can estimate the extent to which water is diffusing unidirectionally as it diffuses more quickly along the length of axonal fibers than it does perpendicular to them (Mori and Zhang, 2006). Measures of fractional anisotropy (FA) are known to follow developmental changes in myelination in the neonate such that FA values get higher as myelin is deposited along axonal fibers in a spatially predictable way (Partridge et al., 2004). Animal studies indicate that both myelinated and nonmyelinated fibers show more anisotropic diffusion properties than gray matter, and suggest that FA is sensitive to both myelin and axonal integrity (Beaulieu and Allen, 1994; Beaulieu, 2002). FA values in gray matter are quite low $(\sim 0.20$ and lower) (Mori and Zhang, 2006), but it is unclear whether nonmyelinated fibers can be distinguished from "gray matter" in humans. The goal of this study was to map group differences in white matter integrity in children and adolescents with FASDs compared with typically developing individuals, and to evaluate the extent to which these abnormalities were related to cognitive impairment observed in FASDs. Although measures of FA reflect the quality or organization of white matter microstructure, we also evaluated group differences in WMD to determine the effects of prenatal alcohol exposure on the location and amount of white matter. Given that findings of gray matter "thickening" in posterior temporal and inferior parietal cortices bilaterally in individuals exposed to alcohol prenatally could result from undermyelination of fibers in these regions, we predicted lower FA and WMD values in the posterior temporal and inferior parietal cortices. Given our own findings of callosal dysmorphology localized to the posterior isthmus and splenium regions (Sowell et al., 2001a), and given findings of lower FA in the isthumus of the corpus callosum by another group (Wozniak et al., 2006), we also expected lower FA and WMD values in the FASD relative to control groups in more posterior regions of the corpus callosum than anterior. Neurocognitive performance within the FASD group was predicted to correlate with white matter abnormalities such that lower FA or WMD values would be associated with worse scores on neurocognitive tests in regions where group differences were observed.

\section{Materials and Methods}

Subjects

Alcohol-exposed subjects. Seventeen children and adolescents with heavy prenatal alcohol exposure who were between the ages of 7 and 15 years underwent brain imaging and neuropsychological evaluation at the University of California, Los Angeles (UCLA). Details of diagnostic procedures are described in a previous report (O'Connor et al., 2006). Briefly, an experienced clinician examined alcohol-exposed children using the Diagnostic Guide for Fetal Alcohol Syndrome (FAS) and Related Conditions (Astley, 2004). This system uses a four-digit diagnostic code reflecting the magnitude of expression of four key diagnostic features of FAS: (1) growth deficiency; (2) the FAS facial phenotype, including short palpebral fissures, flat philtrum, and thin upper lip; (3) CNS dysfunction; and (4) gestational alcohol exposure. All of the children had histories of heavy prenatal alcohol exposure confirmed by maternal report, reliable collateral report, and/or medical or legal records. Using these criteria, four of the children were diagnosed with FAS, five with partial FAS, and seven with alcohol-related neurodevelopmental disorder. One child did not have the facial exam, but met growth, CNS and gestational alcohol exposure criteria to meet diagnostic criteria for alcohol-related neurodevelopmental disorder, at the very least. Because analyses in this report collapse across all FASDs, and this child did meet criteria for an FASD, we did not exclude this child because of the lack of data on facial morphology. Specifics about the amount of alcohol consumed by their mothers were not available, but all 17 had their children removed from their custody as a result of alcohol abuse according to adoptive parents interviewed for the purposes of this study. Some of the children were exposed to other drugs in utero (e.g., marijuana, tobacco), but in all cases, alcohol was reported to be the primary drug of abuse.

Controls. A total of 19 typically developing children between 7 and 15 years of age were studied as a control group. All subjects were screened for neurological impairments, psychiatric illness, history of learning disability, or developmental delay. Control subjects were excluded if they were prenatally exposed to one drink or more per week, or more than two drinks on any one occasion as reported by parents. None of the mothers of control subjects endorsed use of any other illicit drugs during pregnancy. All subjects and their parents gave written assent/consent according to procedures approved by the UCLA institutional review board.

Demographics and alcohol-exposed/control group comparisons for neurocognitive tests can be seen in Table 1 .

\section{Behavioral data}

Subjects were administered a comprehensive neuropsychological battery including measures of intelligence [Wechsler Intelligence Scale for Chil- 
dren, fourth edition (WISC-IV) (Wechsler, 2003)], language, memory, executive function, motor, and visual-spatial functioning. Specific neuropsychological tests chosen to target the functionality of brain regions implicated in the white matter integrity analyses reported here included the test of visuomotor integration (VMI) (Beery, 1989), and reading scores on the Wide Range Test of Achievement (WRAT-RE) (Wilkinson, 1993). The VMI measures visual-motor expression and visual construction. It requires the child to copy increasingly difficult geometric forms. The WRAT is a measure of academic ability, and the reading subtest requires children to read single words of increasing difficulty. One subject with FASD was not administered enough WISC-IV subtests to estimate full scale IQ (FSIQ), although their score for the verbal comprehension index was within the lower end of the range for the FASD group. Group FSIQ means in Table 1 represent scores for the remaining 16 subjects. Handedness was calculated using the Edinburgh Handedness Inventory (Oldfield, 1971) with scores between -100 (completely left handed) and 100 (completely right handed).

\section{MRI scan acquisition}

Both DTI and high-resolution T1-weighted series were collected on a Siemens (Erlangen, Germany) 1.5T Sonata system.

\section{DTI}

Whole-brain DTI data were collected with diffusion weighting gradients applied in six noncollinear directions including a minimum of two and a maximum of four averages per subject $(3 \times 3 \times 3 \mathrm{~mm}, b$ values, 0 and $1000 \mathrm{~s} / \mathrm{mm} 2,50$ axial brain slices covering the entire brain). The signalto-noise ratio (SNR) for white matter (within a region of interest in the genu of the corpus callosum) was calculated for each subject. Within the FASD group, mean SNR values in white matter for the nondiffusion weighted and $b=1000$ images were 11.1 (SD, 4.915) and 3.991 (SD, 0.823 ) respectively. Within the control group, mean white matter SNR values were 11.249 (SD, 2.69) within the nondiffusion weighted image and $3.48(\mathrm{SD}, 0.09)$ in the $b=1000$ image. Group differences on these measures were not significant.

\section{T1-weighted series}

High-resolution T1-weighted sagittal volumes were collected using a three-dimensional magnetization-prepared rapid acquisition gradient echo protocol with the following parameters: repetition time, $1900 \mathrm{~ms}$; echo time, $4.38 \mathrm{~ms}$; flip angle, $15^{\circ}$; matrix size, $256 \times 256 \times 160$; field of view, $256 \mathrm{~mm}$; voxel size, $1 \times 1 \times 1 \mathrm{~mm}$; acquisition time, $8 \mathrm{~min} 8 \mathrm{~s}$. Two to four acquisitions were acquired for each subject. Raters blind to subject age, sex, and diagnosis evaluated image quality, and data from at least two acquisitions were averaged to enhance signal-to-noise ratio.

\section{Image and statistical analysis}

Image preprocessing. To obtain whole-brain and white matter masks, T1-weighted images were preprocessed with a series of manual and automated procedures executed by analysts blind to subject age, sex, and diagnosis. Extracortical tissue was removed from the high-resolution structural MR data using automated brain skull extraction from Brainsuite software (Shattuck et al., 2001; Shattuck and Leahy, 2002). Small errors in the automated processing were manually corrected. The wholebrain masks created were also used to remove extracortical tissue from the diffusion data. T1-weighted images were classified into gray matter, white matter, and CSF (Shattuck et al., 2001) and white matter masks for each subject were created for limiting the search area in the DTI region of interest analyses described below.

For the DTI data, six-parameter rigid body registration was used to align directional and repeat DTI images within subjects. That is, each directional diffusion image was registered to its repeat images using automated image registration (Woods et al., 1998a,b), and each resultant average directional image was merged together into one file using FSL's software library [FSL; Oxford Centre for Functional Magnetic Resonance Imaging of the Brain (FMRIB)'s software library, Oxford University, Oxford, UK; www.fmrib.ox.ac.uk/fsl]. Using FMRIB's Diffusion Toolbox (FDT), warping was applied to the diffusion-weighted images to correct for eddy current distortions (Haselgrove and Moore, 1996), and FA maps were generated for each subject. The FA maps were transformed into the International Consortium for Brain Mapping (ICBM) 305 (Mazziotta et al., 2001) space in the following manner. FSL's FMRIB linear registration tool was used to align each subject's non-diffusionweighted image to the native space high-resolution T1-weighted structural image using a six-parameter registration. The T1 structural image was registered into ICBM 305 space using 12-parameter affine registrations. These two transformations were then applied to each subject's FA map.

FA statistical mapping. Spatially normalized FA images created with FSL were then imported into SPM5 (Wellcome Trust Centre for Neuroimaging, London, UK) and smoothed with a Gaussian kernel of $8 \mathrm{~mm}$ full-width at half maximum. A masking threshold was applied to include only voxels that exceed an FA value of 0.20 in each subject to exclude gray matter voxels and reduce the search area for statistical mapping. An unpaired two-sample $t$ test comparing the spatially normalized smoothed FA maps of controls versus FASD subjects was computed using SPM5. Analyses were corrected for multiple comparisons using false discovery rate with a $p$ value of 0.05 . These analyses allowed us to localize group differences in the diffusion properties of molecules within white matter structures.

WMD statistical mapping. Given that high-resolution T1-weighted image data sets were available for each subject, we were able to use the tissue segmentations from those data to evaluate differences in white matter distribution between groups. We used voxel-based-morphometric (VBM) methods described in numerous previous studies (Paus et al., 1999; Ashburner and Friston, 2000; Sowell et al., 2001b) to localize regions in the brain that contained more or less white matter in the FASD group relative to the controls. VBM allows for a voxel-by-voxel assessment of differences in white matter distribution between groups, and we refer to these WMD differences as differences in white matter macrostructure. Essentially, these analyses allowed us to evaluate differences in brain morphology (i.e., white matter macrostructure) as opposed to the FA analyses that allowed us to measure differences in white matter diffusion characteristics (i.e., white matter microstructure). Spatially normalized white matter masks for each subject were imported into SPM5 and two-sample $t$ tests were computed to determine group differences in white matter density. A masking threshold with a value of 0.01 was applied to exclude nonbrain voxels. Analyses were corrected for multiple comparisons using False Discovery Rate with a $p$ value of 0.05 .

\section{Post hoc FA ROI analyses}

To ensure that group differences in FA were not caused by misregistration of white matter between groups, we evaluated FA values within ROIs mapped to each participants' unsmoothed spatially normalized FA volume in which we had masked out non-white matter voxels (based on segmentation conducted on the high resolution T1-weighted volume). We identified five coordinates chosen from regions of significant group difference in the FA statistical maps described in the results section below (two coordinates in the right temporal lobe, one in each hemisphere from the splenium cluster, and one from a brainstem cluster). Because our a priori hypotheses involved temporal white matter in both hemispheres, we chose two coordinates in the left temporal lobe that were identical to their right hemisphere counterparts (by inverting the left-right component of the right hemisphere temporal lobe coordinates). This would allow us to evaluate for subthreshold effects in the left temporal region that were not significant in the statistical mapping analyses. We located all seven coordinates in the unsmoothed FA white matter masked volumes and calculated the average FA value of all nonzero voxels within an $8 \mathrm{~mm}$ sphere attached to that coordinate for each individual. Twosample $t$ tests were conducted to evaluate the significance of average FA differences between groups within each ROI. This analysis ensured that we were looking at only white matter FA values for conducting statistical analyses and validated the results in the statistical maps.

\section{Brain-behavior correlations}

Simple correlations between raw scores on each of two cognitive tests, VMI and WRAT-RE, were conducted for FA and WMD within each of the 7 ROIs described above. These tests were chosen because they were thought to be from dissociable cognitive domains (visuomotor and read- 
ing), and because they were functions typically attributed to regions where group FA differences were significant in the statistical maps described in the results section below (i.e., VMI for lateral splenial/parietal and WRAT-RE for temporal language regions). We expected VMI scores to correlate with splenial/parietal regions and not temporal regions, and we expected WRAT-RE scores to correlate with temporal but not splenial/parietal regions. Brainbehavior correlations were conducted within each group separately given that group differences on these scores were significant. Where correlations between the brain and behavior were significant, simultaneous multiple regression using group, score and group-by-score interactions to predict FA or WMD values were also conducted to evaluate the extent to which correlations between brain and behavior statistically differed between groups.

\section{Results}

Demographic and behavioral measures As shown in Table 1, FASD and control groups were well matched on gender, age handedness, and maternal education (of the adoptive mothers for the FASD group and biological mothers for the nonexposed) with no significant group differences on these measures. As would be expected, control subjects performed significantly better on tests of general intellectual functioning (estimated FSIQ from the WISC-IV), visuomotor integration (VMI), and reading ability (WRAT-RE). Furthermore, there were fewer Caucasian subjects in the control than the FASD group.

\section{Statistical mapping}

ICBM coordinates, $p$ values and cluster volumes for significant clusters for the FA and WMD VBM analyses are shown in Table 2. Statistical maps of FA group differences, WMD differences, and their spatial overlap are shown in Figure 1.

\section{FA}

As seen in dark blue (FA group differences only) and light blue (FA and WMD group differences overlap) in Figure 1, the most notable significant clusters (FDR corrected for multiple comparisons, $p<0.05$ ) where FA was significantly lower in the FASD relative to control subjects fell in regions of the lateral splenium (medial superior parietal white matter) and posterior cingulate white matter bilaterally and in the deep white matter of the right temporal lobe. The temporal lobe clusters included portions of the inferior association fibers that connect the occipital to temporal and frontal lobes (i.e., inferior longitudinal fasciculus, inferior fronto-occipital fasciculus) (Wakana et al., 2004). Other significant clusters where FA was lower in the FASD group were observed in right internal capsule and brainstem regions. There were no regions where FA was significantly higher in the FASD relative to control subjects.
Table 2. Results from SPM analyses of group differences in FA and WMD

\begin{tabular}{|c|c|c|c|}
\hline Cluster & $p$ & $\begin{array}{l}\text { Cluster size } \\
\left(\text { in } \mathrm{mm}^{3} \text { ) }\right.\end{array}$ & $x, y, z$ coordinates \\
\hline \multicolumn{4}{|c|}{ FDR corrected } \\
\hline \multicolumn{4}{|l|}{ FA (lower FA in FASD) } \\
\hline R temporal lobe & $<0.001$ & 3070 & $\begin{array}{l}45,-23,-15 \\
34,-13,-11 \\
39,-45,0\end{array}$ \\
\hline L LS/parietal white matter & $<0.001$ & 3764 & $\begin{array}{l}-8,-51,22 \\
-6,-35,28\end{array}$ \\
\hline R LS/parietal white matter & SVC correc & & $8,-30,32$ \\
\hline \multicolumn{4}{|c|}{ WMD VBM (lower white matter density in FASD) } \\
\hline $\begin{array}{l}\text { LLS/parietal white matter } \\
\text { R LS/parietal white matter }\end{array}$ & $<0.001$ & 1495 & $\begin{array}{l}-14,-50,15 \\
-4,-43,18 \\
6,-41,19 \\
4,-37,22\end{array}$ \\
\hline
\end{tabular}

$p$ values, cluster volumes, and $x, y$, and $z$ ICBM coordinates for the maximum $z$ value within each cluster for the $\mathrm{FA}(p<0.05$, FDR correction for multiple comparisons) and WMD (voxel threshold $p<0.001$, small volume correction for multiple comparisons) are presented. R, Right; $L$, left; $L S$, lateral splenium.

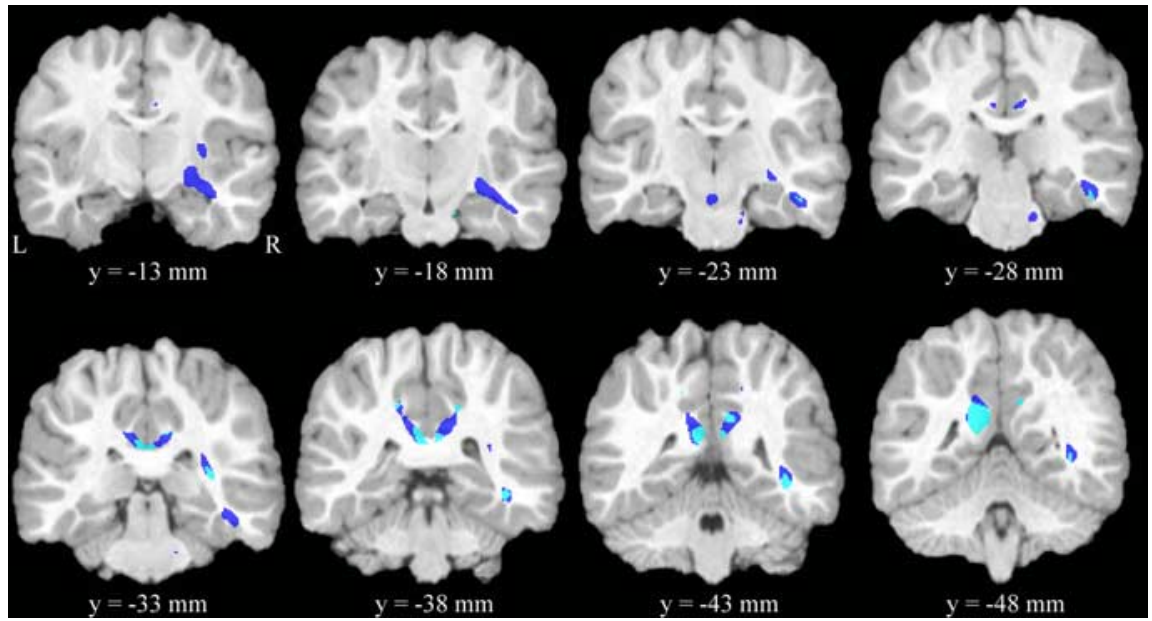

Figure 1. Results from statistical parametric mapping of FA and WMD. Coronal slices of one representative subject's brain with significantly lower in the individuals with FASD relative to control subjects ( $p<0.05$, FDR correction for multiple comparisons). Regions in light blue are regions where WMD was lower in the FASD relative to controls ( $p<0.001$, uncorrected) and overlapped with regions of lower FA in FASD. There were numerous regions where WMD was lower in FASD relative to controls, but they were not within the region of interest used in the small volume correction for multiple comparisons.

\section{White matter density}

There were no regions where white matter density group differences passed correction for multiple comparisons using FDR with $p<0.05$ (FASD $>$ control or control $>$ FASD). Because we were also interested in WMD to help explain differences in FA (i.e., microstructural organization) from group differences in white matter morphology (i.e., macrostructural organization), we examined uncorrected (extent threshold $=0, p<0.001$ ) group differences in WMD within regions of interest that were significant in the FA group analyses. As seen in Figure 1 in light blue (WMD and FA group differences overlapping), WMD was lower in the FASD group in some, but not all regions where FA was affected. Specifically, lower WMD was observed in the lateral splenium of the corpus callosum bilaterally and in right deep temporal association fibers. Notably, little overlap between FA and WMD differences were observed in the more anterior portions of the right temporal lobe and more lateral aspects of the splenium where only FA differences were observed. Small volume correction (SVC) for multiple comparisons within the region of interest that included only significant voxels from the FA analyses 
Table 3. Post hoc region of interest FA analyses

\begin{tabular}{|c|c|c|c|c|c|c|}
\hline \multirow[b]{2}{*}{ Region } & \multicolumn{2}{|l|}{ FASD } & \multicolumn{2}{|l|}{ Control } & \multirow[b]{2}{*}{$t$} & \multirow[b]{2}{*}{$p$} \\
\hline & Mean FA & SD & Mean FA & SD & & \\
\hline R ant temp & 0.355 & 0.021 & 0.385 & 0.024 & 4.039 & $<0.001$ \\
\hline R post temp & 0.408 & 0.046 & 0.451 & 0.030 & 3.249 & 0.003 \\
\hline L splenium & 0.473 & 0.100 & 0.565 & 0.047 & 3.461 & 0.002 \\
\hline R splenium & 0.472 & 0.081 & 0.553 & 0.050 & 3.544 & 0.002 \\
\hline Brainstem & 0.377 & 0.027 & 0.408 & 0.037 & 2.890 & 0.007 \\
\hline Lant temp & 0.382 & 0.033 & 0.403 & 0.034 & 1.828 & 0.076 \\
\hline L post temp & 0.423 & 0.028 & 0.442 & 0.036 & 1.740 & 0.091 \\
\hline
\end{tabular}

Regions of interest defined based on the splenium and temporal lobe clusters observed in statistical maps. R, Right; L, left; ant, anterior; post, posterior; temp, temporal lobe.

\section{Lateral splenium Right temporal lobe}

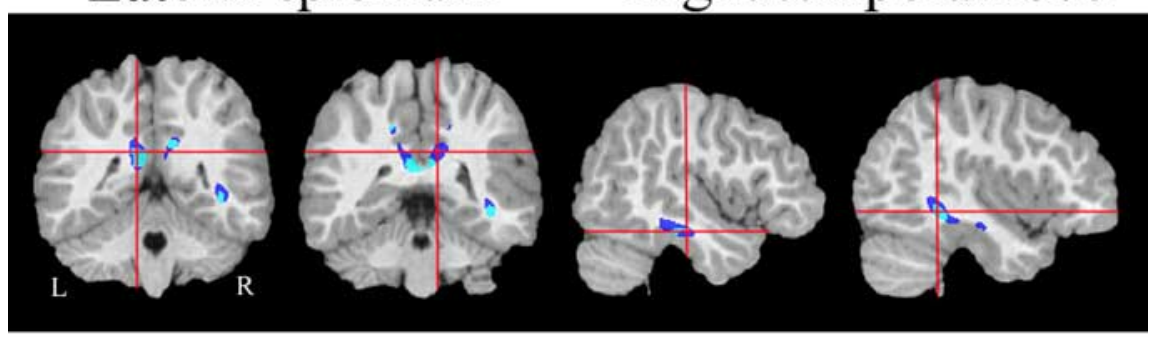

$(-9,-45,22)$

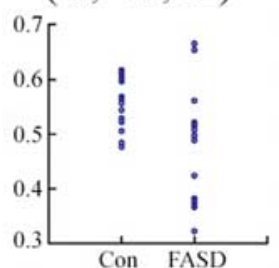

$(11,-41,27)$

$(45,-22,-15)$
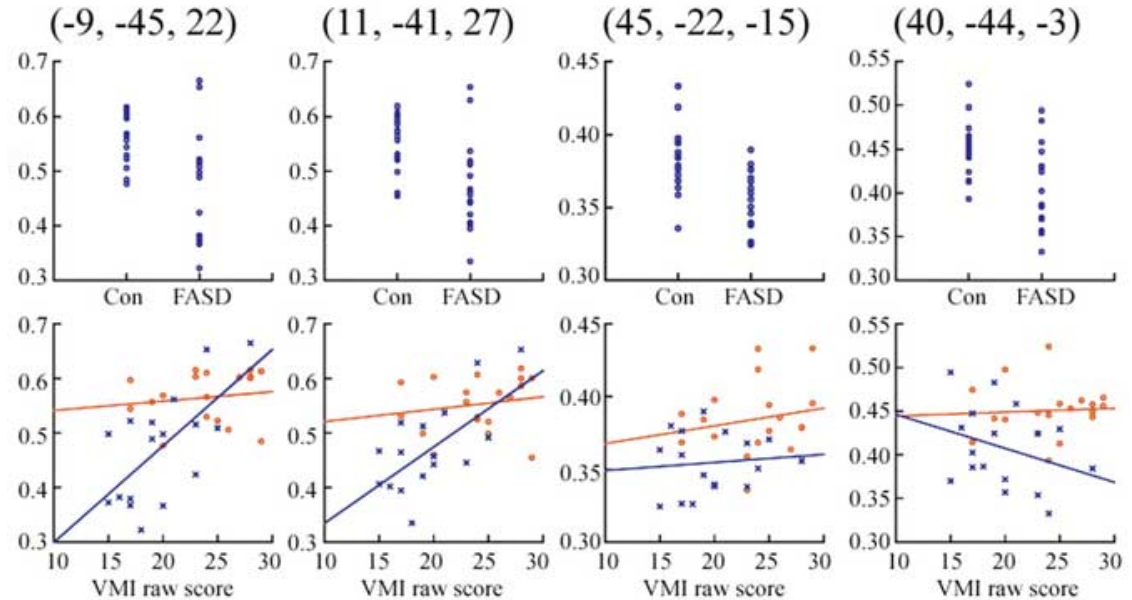

Figure 2. ROIFA analyses and brain behavior correlations. Coronal and sagittal slices of one representative subject's brain with significant clusters from the FA and WMD analyses. Color coding for significant clusters is the same as described for Figure 1. The $y$-axes for all graphs have FA values. In the top row of graphs, FA values for each subject are shown for the FASD and control groups. FA values were taken from ROIs (localized approximately at the intersection of the crosshairs in each image) of all voxels within an $8 \mathrm{~mm}$ sphere in the unsmoothed, white matter masked FA volume for each individual. In the bottom row, FA values from the same ROIs are shown in relation to subjects behavioral scores on the VMI. Control subjects are shown in red and FASD subjects in blue. Correlations between VMI scores and FA were highly significant in the left and right lateral splenium ROIs within the FASD, but not control subjects, and not for either group in the anterior and posterior temporal ROIs. seen in Table 3, the variance in the splenium ROIs was considerably higher than the variance in other regions, although it is not clear why. The increased variance was seen in both groups, however, and was unlikely to impact the significant group effects. ROIs from the left temporal lobe were not above the statistical threshold used to create the map, but were actually at trend level significance in the ROI analysis as shown in Table 3.

\section{Brain-behavior results}

Results from the brain behavior correlation analyses are illustrated in the bottom row of graphs in Figure 2. As predicted, correlations between VMI scores and FA values in the lateral splenium-parietal white matter ROIs bilaterally were significant, but only within the FASD group (left, $r=$ 0.653, $p=0.006$; right, $r=0.681, p=$ $0.004)$. Essentially in these regions within the FASD group, lower FA values were predictive of lower VMI scores. Group (FASD vs control) by score (VMI) interactions in multiple regression analyses suggest that the correlation between splenium FA and VMI scores differed significantly between groups (left, $\beta=1.86, p=0.009$; right, $\beta=$ $1.57, p=0.031)$. Although we expected the reading score to be correlated with temporal lobe FA, correlations between the WRAT-RE score and FA were not significant in any region tested. Correlations between WMD and either VMI or WRAT-RE were not significant for any of the seven ROIs.

confirmed three significant clusters: one in the left temporal lobe and one in each the left and right lateral splenium/parietal regions (Table 2). There were no regions where WMD was higher in the FASD group relative to controls within the region of interest.

\section{Post hoc FA ROI analyses}

Results from post hoc FA white-matter ROI analyses are shown in Table 3 and Figure 2. FA differences between groups were highly significant in five of the seven ROIs evaluated. Scatterplots of group effects are shown in the top row of graphs in Figure 2 and are mapped from the two splenium and two right temporal lobe ROIs. These significant results further validated the statistical maps, and showed that FA differences seen in the maps cannot be attributed to misregistration of white matter between groups. As

\section{Discussion}

These findings further confirm white matter pathology associated with heavy prenatal alcohol exposure. The regional findings are in part consistent with our a priori hypotheses in that we observed lower FA in the FASD than control groups in lateral temporal regions and in the vicinity of the lateral splenium of the corpus callosum where we had previously observed dysmorphology in independent samples (Sowell et al., 2001a, 2008). Our findings are somewhat consistent with previous reports of FA differences in FASD in posterior callosal regions (Ma et al., 2005; Wozniak et al., 2006) as we found lower FA values localized to more posterior than anterior perisplenial white matter. Previous studies of FA in FASD have evaluated only midsagittal corpus 
callosum regions of interest, and not whole-brain voxel-based analyses of FA as we have conducted here. Thus, our findings of lower FA values in ILF regions are novel. Results from this study support the hypothesis that higher gray matter thickness in individuals with FASDs in posterior temporal and inferior parietal lobes observed in previous reports (Sowell et al., 2008) could result from delayed or permanently reduced myelin deposition, at least in the right temporal lobe. In other words, it is possible that tissue with "gray matter" signal on MRI is actually unmyelinated peripheral axonal or dendritic fibers. It is also possible that disorganization of white matter fibers (i.e., fibers with multiple orientations within the same voxel), or lower axonal density could account for the lower FA (Mori and Zhang, 2006) we observe in the FASD group. It is not clear, however, how disorganization or reduced axonal density of myelinated fibers (which would likely have white matter signal on T1-weighted MRI) could result in higher colocal gray matter thickness values, further implicating dysmyelination rather than disorganization as a cause. Animal or postmortem studies would help further explain the etiology of lower FA and higher cortical thickness associated with heavy prenatal alcohol exposure.

Whatever the microstructural (evaluated with FA analyses) or macrostructural (evaluated with WMD analyses) etiology, abnormalities of FA in some brain regions of the FASD subjects are related to impairment in cognitive functions subserved by those regions. Specifically, lower FA in the lateral splenium and parietal lobe white matter predicted worse performance on a test of visuomotor integration in the FASD group. This finding was specific to FA (and not WMD) in the lateral splenium region, as would be expected given that regions role in visuospatial processing $(\mathrm{Ca}-$ beza and Nyberg, 2000), specific to the FASD group, and specific to visuomotor integration (i.e., reading ability was not related to FA values in the splenium region). Thus, these white matter microstructural (i.e., reduced myelination or fiber disorganization) abnormalities in children and adolescents with heavy prenatal alcohol exposure appear to be clinically significant. Our results suggest that this region of white matter is particularly susceptible to damage from prenatal alcohol exposure and that FA in this region may not be variable enough to correlate with VMI performance in the controls. It is also likely that other brain regions not measured in these analyses are related to visuomotor integration in either or both groups.

We also conducted VBM analyses of WMD to evaluate macrostructural white matter differences. Although WMD results were not significant after correction for multiple comparisons, results from the uncorrected analyses suggest a trend toward lower WMD in bilateral lateral splenial and right temporal lobe regions. Lower WMD overlapped with some, but not all voxels significant in the FA analyses. Thus, FA differences between groups cannot be completely mediated by morphological differences in white matter (i.e., comparing white matter tissue in one group to gray matter tissue in another), and must be related to lower myelination or disorganization of white matter fibers. Decreased WMD could also be related to the increased cortical thickness in temporal lobe regions reported previously (Sowell et al., 2008) given that unmyelinated fibers within the periphery of the cortex may appear as "gray matter" on T1-weighted MRI and may have been included in our gray matter thickness measure in the previous report. Future analyses could involve fiber tractography (Mori and Zhang, 2006) to define exact fiber tracts within the temporal lobe (i.e., inferior longitudinal fasciculus) in each individual, and group differences in FA within matched tracts could be evaluated (Johansen-Berg and Behrens, 2006). This type of analysis would help explain observed FA differences caused by mismatched anatomy between groups, and could reveal actual differences in myelination or fiber organization at homologous anatomical structures.

Interestingly, we did not observe wider spread lower WMD measures in the lateral aspects of the temporal lobes in the left hemisphere as we did in a previous report (Sowell et al., 2001b). It is possible that WMD reductions in the left temporal lobe were subthreshold and at trend level significance in this sample, just as lower FA was in the ROI analyses. It is also possible that differences between the two independent samples accounted for the discrepant results. The previous group we studied tended to be more severely affected than those in the current report with 14 of 21 subjects in the earlier sample having the full FAS diagnosis (Sowell et al., 2001b) and only 4 of 17 meeting full FAS diagnostic criteria in the current report. We have shown that children with heavy prenatal alcohol exposure but without the facial dysmorphology to obtain the FAS diagnosis have regionally similar, but less severe brain dysmorphology (Sowell et al., 2001a,b, 2008; O'Hare et al., 2005). Perhaps the lower proportion of individuals with the full FAS diagnosis in the current report is weakening the observed effects. Unfortunately, with only 4 subjects meeting full FAS diagnostic criteria in this group, statistical power was not sufficient to explain severity effects in the present sample.

We did not confirm lower FA values in the left temporal lobe in the voxel-based analyses. We would have expected similar effects in the left and right hemispheres given bilateral findings of higher gray matter thickness in an independent sample of alcohol-exposed subjects (Sowell et al., 2008). The post hoc ROI analyses revealed trend level differences, with lower FA values in the left temporal lobe in the subjects with FASDs. This suggests that greater thickness in the left hemisphere may also be related to disorganization or reduced myelin, although not to the extent that we see in the right hemisphere where FA decreases were statistically significant.

Together, the results presented here strongly implicate white matter microstructural abnormalities in children and adolescents with FASDs, exclusive of relative differences in white matter morphology. Lower FA in the FASD group in lateral splenial/parietal white matter are related to poorer visuomotor integration, although cause-and-effect cannot be derived from such correlational analyses. Whether myelin deposition and organization of white matter fibers is required for intact visuomotor integration, or experience and facility at visuomotor integration result in higher deposition of myelin and organization of fibers is difficult to infer from human studies. Studies of normal childhood and adolescent development show regionally heterogeneous declining cortical thickness, presumably caused by synaptic pruning and greater myelination (for review, see Sowell et al., 2004). Longitudinal analyses could help in understanding the cascade of events that result in lower FA and WMD in individuals with heavy prenatal alcohol exposure, and whether the insults to the brain are static, or plastic throughout the years of development. Animal studies could also help determine the etiology of white matter abnormalities resultant from prenatal alcohol exposure. Further caveats are warranted regarding difficulties in determining causality of prenatal alcohol exposure in the observed group effects on brain structure and cognitive function given that this is a case-control study and it was not possible to control many other potentially significant differences between the groups (i.e., socioeconomic status among the biological parents of participants with FASDs, prenatal exposure to other drugs in the FASD 
group) that could be associated with brain and cognitive differences between groups.

\section{References}

Archibald SL, Fennema-Notestine C, Gamst A, Riley EP, Mattson SN, Jernigan TL (2001) Brain dysmorphology in individuals with severe prenatal alcohol exposure. Dev Med Child Neurol 43:148-154.

Ashburner J, Friston KJ (2000) Voxel-based morphometry: the methods. NeuroImage 11:805-821.

Astley SJ (2004) Diagnostic guide for fetal alcohol spectrum disorders: the 4-digit diagnostic code. Seattle: Washington UP.

Beaulieu C (2002) The basis of anisotropic water diffusion in the nervous system - a technical review. NMR Biomed 15:435-455.

Beaulieu C, Allen PS (1994) Determinants of anisotropic water diffusion in nerves. Magn Reson Med 31:394-400.

Beery KE (1989) Manual for the developmental test of visual-motor integration. Cleveland: Modern Curriculum.

Bookstein FL, Sampson PD, Streissguth AP, Connor PD (2001) Geometric morphometrics of corpus callosum and subcortical structures in the fetalalcohol-affected brain. Teratology 64:4-32.

Bookstein FL, Streissguth AP, Sampson PD, Connor PD, Barr HM (2002) Corpus callosum shape and neuropsychological deficits in adult males with heavy fetal alcohol exposure. NeuroImage 15:233-251.

Cabeza R, Nyberg L (2000) Imaging cognition II: an empirical review of 275 PET and fMRI studies. J Cogn Neurosci 12:1-47.

Haselgrove JC, Moore JR (1996) Correction for distortion of echo-planar images used to calculate the apparent diffusion coefficient. Magn Reson Med 36:960-964.

Johansen-Berg H, Behrens TE (2006) Just pretty pictures? What diffusion tractography can add in clinical neuroscience. Curr Opin Neurol 19:379-385.

Jones KL, Smith DW (1973) Recognition of the fetal alcohol syndrome in early infancy. Lancet 2:999-1001.

Jones KL, Smith DW (1975) The fetal alcohol syndrome. Teratology 12:1-10.

Ma X, Coles CD, Lynch ME, Laconte SM, Zurkiya O, Wang D, Hu X (2005) Evaluation of corpus callosum anisotropy in young adults with fetal alcohol syndrome according to diffusion tensor imaging. Alcohol Clin Exp Res 29:1214-1222.

Mattson SN, Riley EP (1998) A review of the neurobehavioral deficits in children with fetal alcohol syndrome or prenatal exposure to alcohol. Alcohol Clin Exp Res 22:279-294.

Mattson SN, Riley EP, Sowell ER, Jernigan TL, Sobel DF, Jones KL (1996) A decrease in the size of the basal ganglia in children with fetal alcohol syndrome. Alcohol Clin Exp Res 20:1088-1093.

Mattson SN, Riley EP, Gramling L, Delis DC, Jones KL (1998) Neuropsychological comparison of alcohol-exposed children with or without physical features of fetal alcohol syndrome. Neuropsychology 12:146-153.

Mazziotta J, Toga A, Evans A, Fox P, Lancaster J, Zilles K, Woods R, Paus T, Simpson G, Pike B, Holmes C, Collins L, Thompson P, MacDonald D, Iacoboni M, Schormann T, Amunts K, Palomero-Gallagher N, Geyer S, Parsons L, et al. (2001) A probabilistic atlas and reference system for the human brain: International Consortium for Brain Mapping (ICBM). Philos Trans R Soc Lond B Biol Sci 356:1293-1322.

Mori S, Zhang J (2006) Principles of diffusion tensor imaging and its applications to basic neuroscience research. Neuron 51:527-539.

National Center on Birth Defects and Developmental Disabilities Centers for Disease Control and Prevention Department of Health and Human Services (2004) Fetal alcohol syndrome guidelines for referral and diagnosis: national task force on fetal alcohol syndrome and fetal alcohol effects. Retrieved on January 11, 2008, from http://www.cdc.gov/ncbddd/fas/ documents/FAS_guidelines_accessible.pdf.

O'Connor MJ, Frankel F, Paley B, Schonfeld AM, Carpenter E, Laugeson EA, Marquardt R (2006) A controlled social skills training for children with fetal alcohol spectrum disorders. J Consult Clin Psychol 74:639-648.

O’Hare ED, Kan E, Yoshii J, Mattson SN, Riley EP, Thompson PM, Toga AW,
Sowell ER (2005) Mapping cerebellar vermal morphology and cognitive correlates in prenatal alcohol exposure. NeuroReport 16:1285-1290.

Oldfield RC (1971) The assessment and analysis of handedness: the Edinburgh inventory. Neuropsychologia 9:97-113.

Partridge SC, Mukherjee P, Henry RG, Miller SP, Berman JI, Jin H, Lu Y, Glenn OA, Ferriero DM, Barkovich AJ, Vigneron DB (2004) Diffusion tensor imaging: serial quantitation of white matter tract maturity in premature newborns. NeuroImage 22:1302-1314.

Paus T, Zijdenbos A, Worsley K, Collins DL, Blumenthal J, Giedd JN, Rapoport JL, Evans AC (1999) Structural maturation of neural pathways in children and adolescents: in vivo study. Science 283:1908-1911.

Riikonen R, Salonen I, Partanen K, Verho S (1999) Brain perfusion SPECT and MRI in fetal alcohol syndrome. Dev Med Child Neurol 41:652-659.

Roebuck TM, Mattson SN, Riley EP (1998) A review of the neuroanatomical findings in children with fetal alcohol syndrome or prenatal exposure to alcohol. Alcohol Clin Exp Res 22:339-344.

Roebuck TM, Mattson SN, Riley EP (1999) Behavioral and psychosocial profiles of alcohol-exposed children. Alcohol Clin Exp Res 23:1070-1076.

Shattuck DW, Leahy RM (2002) BrainSuite: an automated cortical surface identification tool. Med Image Anal 6:129-142.

Shattuck DW, Sandor-Leahy SR, Schaper KA, Rottenberg DA, Leahy RM (2001) Magnetic resonance image tissue classification using a partial volume model. NeuroImage 13:856-876.

Sowell ER, Mattson SN, Thompson PM, Jernigan TL, Riley EP, Toga AW (2001a) Mapping callosal morphology and cognitive correlates: effects of heavy prenatal alcohol exposure. Neurology 57:235-244.

Sowell ER, Thompson PM, Mattson SN, Tessner KD, Jernigan TL, Riley EP, Toga AW (2001b) Voxel-based morphometric analyses of the brain in children and adolescents prenatally exposed to alcohol. NeuroReport 12:515-523.

Sowell ER, Thompson PM, Mattson SN, Tessner KD, Jernigan TL, Riley EP, Toga AW (2002a) Regional brain shape abnormalities persist into adolescence after heavy prenatal alcohol exposure. Cereb Cortex 12:856-865.

Sowell ER, Thompson PM, Peterson BS, Mattson SN, Welcome SE, Henkenius AL, Riley EP, Jernigan TL, Toga AW (2002b) Mapping cortical gray matter asymmetry patterns in adolescents with heavy prenatal alcohol exposure. NeuroImage 17:1807-1819.

Sowell ER, Thompson PM, Toga AW (2004) Mapping changes in the human cortex throughout the span of life. Neuroscientist 10:372-392.

Sowell ER, Lu LH, O'Hare ED, McCourt S, Mattson SN, O'Connor MJ, Bookheimer SY (2007) Medial temporal and frontal lobe activation abnormalities during verbal learning in children with fetal alcohol spectrum disorders. NeuroReport 18:635-639.

Sowell ER, Mattson SN, Kan E, Thompson PM, Riley EP, Toga AW (2008) Abnormal cortical thickness and brain-behavior correlation patterns in individuals with heavy prenatal alcohol exposure. Cereb Cortex 18:136-144.

Swayze VW, Johnson VP, Hanson JW, Piven J, Sato Y, Giedd JN, Mosnik D, Andreasen NC (1997) Magnetic resonance imaging of brain anomalies in fetal alcohol syndrome. Pediatrics 99:232-240.

Wakana S, Jiang H, Nagae-Poetscher LM, van Zijl PC, Mori S (2004) Fiber tract-based atlas of human white matter anatomy. Radiology 230:77-87.

Wechsler D (2003) The Wechsler Intelligence Scale for Children, Ed 4. San Antonio, TX: The Psychological Corporation.

Wilkinson GS (1993) Manual for The Wide Range Achievement Test, Ed 3. Wilmington, DE: Wide Range.

Woods RP, Grafton ST, Holmes CJ, Cherry SR, Mazziotta JC (1998a) Automated image registration: I. General methods and intrasubject, intramodality validation. J Comput Assist Tomogr 22:139-152.

Woods RP, Grafton ST, Watson JD, Sicotte NL, Mazziotta JC (1998b) Automated image registration: II. Intersubject validation of linear and nonlinear models. J Comput Assist Tomogr 22:153-165.

Wozniak JR, Mueller BA, Chang PN, Muetzel RL, Caros L, Lim KO (2006) Diffusion tensor imaging in children with fetal alcohol spectrum disorders. Alcohol Clin Exp Res 30:1799-1806. 